\title{
AUTOMATED OPTICAL TESTING
}

\author{
H.J. Tiziani, Institute of Applied Optics, \\ University of Stuttgart, \\ Pfaffemwaldring 9, 7000 Stuttgart 80 , West Germany
}

\section{INTRODUCTION}

Interferometry, holographic interferometry and speckle techniques are beconing useful tools for precision measurements in research and for industrial applications. Computer analysis ist increasingly important for the fringe analysis. The use of solid state detector-arrays, image menory boards together with microprocessors and computers for the extraction of the information from the interferograms and high resolution graphic boards find important application in optical metrology. Much more information can be extracted from the interferograms leading to higher sensitivities and accuracies.

Automated quantitative evaluation of interferograms requires accurate interference phase measurements, independent of fringe position and intensify, variations superposed onto the interferograms. In many interferometric arrangements, phase shifting , heterodyne by phase locked (5) techniques have been introduced for automatic fringe analysis. In the phase shifting technique or quasi-heterodyne technique the relative phase is changed continuously or stepwise, using at least three phase shifts of 90 or 120 degrees. The phase of the interference patterns can then be computed from the different peasured intensity values. The phase shifting technique is very approprlate for digital processing and TV techniques. Interferometry and two reference beamholography together with video electronic processing lead to a sensitivity of $1 / 100$ of a fringe at any point of the fringe pattern in the TV image. In heterodyne methods the relative phase increases ifnearly in time and the reference phase is measured electronically at the beat frequency of the reconstructed waveffelds. Heterodyne interferometry and holographic interferometry offer high spatial resolution and interpolation up to $1 / 1000$ of a fringe. It requires, however, sophisticated electronic equipment and mechanical scanning of the fringe pattern. An automatic fringe analysis leads to the wavefront. Polynoms are very useful for error as well as for system analysis.

For engineering applications real time techniques in interferometry, holographic interferometry as well as for speckle applications are desired. Thermoplastic material is used frequently for the hologram storage in engineering applications. Photorefractive crystals and thin films such as of amorphous arsenic trisulfide are found to be useful for real time holography and speckle applications.

\section{INTERFEROMETRIC TESTING}

Solid state detector arrays, and microprocessors are mostly responsible for the progress in interferometric testing being made during the last few years. Digital interferometry provides means for obtaining very precise measurements at rapid rates.

For the fringe analysis many different methods are applied. They can be classiffed into static and dynamic methods. In static methods a tilt is introduced to avoid closed fringes. The fringe centers can be found manually and by using a digitizing tablet as well as by using video- and image processing teghniques. Furthermore, phase detection technique in the spatial domain using Fourier transformation to or Fourier analysis in connection with video technique can be used.

The intensity of the interference pattern can be written as

$$
I(x, y)=I_{0}(x, y)\{1+b(x, y) \cdot \cos [\phi(x, y)+\Delta]\}
$$

where $I_{0}$ is the average intensity at each detector point, $b$ is the modulation of the fringe pattern.

$$
\phi(x, y)=\frac{2 \pi}{\lambda} w(x, y) \text { is the phase distribution of the wavefront } w(x, y) \text { across the interference }
$$

pattern to be measured.

For dynamic algorithms the relative phase between the reference beam and the test beam in an interferometer is varfed by at constant, controlled rate or in steps.

The interference pattern can be recorded by a solid state detector array. For the shifting technique at least three patterns with the appropriate phase shifts need to be recorded.

Applying four phase shifts $\left(-\frac{3 \pi}{4},-\frac{\pi}{4}, \frac{\pi}{4}, \frac{3 \pi}{4}\right)$ the phase of the wavefront computed from the four interferograms is:

$$
\phi(x, y)=\tan ^{-1}\left[\frac{I_{4}(x, y)-I_{2}(x, y)}{I_{1}(x, y)-I_{3}(x, y)}\right]
$$


For accurate phase analysis, the phase shifts introduced by a piezo element for instance need to be calibrated. An error of $\lambda / 10$ in the wavefront occurs by an error in the phase of 20 per cent. Care needs to be taken when phase shifts in strongly diverging or converging beams are necessary.

Very often it is useful to describe the wavefront by polynomials. Zernike polynomials are useful because of their orthogonal properties. For special applications Zernike-Tatian-polynomials (for systems with central obstruction) or Tschebyscheff polynomials are useful.

For the characterisation of extremely smooth surfaces total integrated and angle-resolved scattering, stylus profilometry and heterodyne profilometry have been used. Scattering methods lead to statistical information about the surface and are noncontact. By contrast surface damage is risked with contacting stylus profilometers. Heterodyne profilometers can give height resolution in the order of 1 ?

with a lateral resolution 11 mited by diffraction of $1 \mu \mathrm{m}$.
Heterodyne interferometry was exfegsively treated by Dandliker ${ }^{4}$ anong others and applied to heterodyne profilometry recently by Huang and others. Shearfpg and two wavelength technigues are very

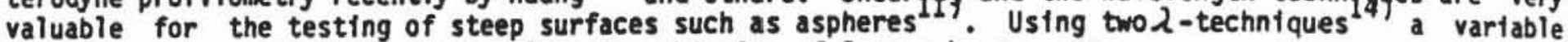
measurement sensitivity is attainable, the ambiguity of $2 \pi$ can be overcome.

\section{COMPONENT AND SYSTEM ANALYSIS FROM INTERFERENCE PATTERNS}

Twyman Green and Fizeau interference arrangements are frequently used for testing spherical and aspherical surfaces, By testing aspherical surfaces using computer generated holograms (CGH) for absolute measurements ${ }^{12}$ ) or by relative measurements using holographic techniques, the asphericity as well as the adjustment errors of the test surface pegd to be determined. Adjustment errors can be evaluated from the interferogran and compensated ${ }^{12}$ 13). By testing aspherical surfaces the coefficients of asphericity and adjustment errors of test surfaces and holograms can be determined from the interferograms. In a combined measuring technique including ray tracing and fringe analysis a procedure for system analysis was developed including the following steps:

- automated fringe analysis

- development of the measured wavefront of the fringe pattern into polynominals

- considering polynom-coefficients as goal for the optimization

- variation of the system parameter to obtain best agreement between measurements and calculations.

At first the wavefront is deterwined applying optical ray tracing techniques leading to a fringe system. Usually a departure of the measured wavefront of the actual system to the computed occurs because the system parameters may be slightly different. The aim is not the optimization of the original systen but the modification of the system parameters in such a way that the measured wavefront $f_{3}$ ) grees with the one computed from the optical data in order to find the parameter errors in the system ${ }^{3}$ ).

Damped least square techniques are useful. The system parameters such as: radius of curvature, refractive index, centring errors are varied to obtain the measured wavefront. Zernike polyhomials are very useful for system analysis. To vary the parameters of the actual system starting with Zernike coefficients $F_{1}$ obtained from the measured fringe pattern we can write:

$$
F_{1}=G_{i}\left(x_{21}-x_{a 1}\right)
$$

were $G_{1}$ is a weighting factor and $x_{3 i}$ and $x_{1}$ are the target and actual values respectively

Expression 3) can be written as 'Tailiop'expansion

$$
F_{1}=F_{01}+\sum_{j=1}^{m} B_{i j}\left(x_{j}-x_{0 j}\right)+\ldots
$$
By variation of the parameter to approach the target $x_{j}$ the departure of the different system parameters
from the theoretical values can be found.

$$
B_{i f}=\frac{\delta P_{1}}{\delta X_{j}} \text { is the sensitivity of the system to variation of parameters found from ray tracing. }
$$

$x_{1}-x_{0}$ can be found by inverting equation 4$)$. Usually the number of equations is larger than the number $8 f$ parameters. Therefore, the merit function should lead to a minimum.

\section{RESULTS AND CONCLUSION}

Some of our applications of component and systen analysis were:

- the analysis of adjustment errors when testing spherical and aspherical surfaces

- determination of the asphericity coefficients and vertex curvature of contact lenses

- fine adjustments of air spacings in optical systems

- measuring of refractive index of the elements of optical systems. 
Adjustment errors occuring by the test procedure modify the fringe pattern. It should be noted that by testing aspherical surfaces with CGH, seven degrees of freedom need to be considered. From the analysis of the fringe pattern the contribution of adjustment errors like centring errors can be calculated and subtracted from the wafefront. Care needs to be taken when subtracting adjustment errors by asymetric surface errors.

An arrangement for testing spherical and aspherical surfaces is shown in fig. 1 . For testing aspherical surfaces, CGH are frequently used. In fig. 1, the auxillary lens Al used for testing spherical surfaces can be replaced by a combination of simple lenses when testing complicated aspheric surfaces in order to adapt the aperture and compensate some asphericity. Furthermore the image of the aspherical surface under test should be near the CGH. For fringe analysis, the phase-shift introduced into the reference beam occurs by means of the piezo-driven mirror $M$. An example of compensation of adjustment errors is shown in fig. 2, where different tilts and centring errors were compensated. The same procedure can be applied to compensate focussing errors. To obtain a quasi microstructure analyses, the shape error of the surface can be subtracted. From the fringe analysis, shape errors as well as aspheric parameters or adjustment errors can be evaluated.

contactlenses will be selected and spectfied by the vertex curvature and the aspherical parameters, wich in turn can be specified by the excentricity of the back surface. The determination of such parameters from the fringe pattern is obtained with the procedure described in equation 4).

In another example a high aperture optical system was analysed. From the design an almost diffraction 1 imited performance was expected. However, due to production errors a rather limited image quality was obtained. With the fringe analysis it could be demonstrated that with the iteration process by change of air spacings the wavefront could be much improved and the system could finally be used. Two air spacings were easily accessible and were modified to obtain the appropriate image quality found after a few fterations. A residual salil zonal error was left. The result is shown in fig. 3.

In fig. 4 by contrast an example of a quasi heterodyne technique for contactless microstructure analysis of a compact disc is shown. With heterodyne-techniques depth resolution of $1 \&$ and better is possible by a lateral resolution of $1 \mu \mathrm{m}$.

Optical metrology combined with automatic fringe analysis lead to powerful tools for many applications in industry to extend the range in microprofile measurements and shape analysis two $\lambda$-techniques in interferometry and holography can be useful in the future.

\section{REFERENCE}

1) B. Dörband, thes1s, University Stuttgart (1986)

2) K. Creath, SPIE 680,19 (1986)

3) B. Breuckmann and W. Thieme, Appl. Opt. 24, 2145 (1985)

4) R. Dändliker, Heterodyne Holographic Interferometry in Progress in Optics Vol. XVII, E. Wolf ed. North Holl and Amsterdam, 1 (1980)

5) W. Volmson, D.C. Leiner and D.T. Moore, Opt. Eng. 18, 46 (1979)

6) Z. Sodnik and H.J. Tiziani, Opt. Comm. 58,295 (ig86)

7) M. Takeda, H. Ina and S. Kobayashí, J. Opt. Soc. Am. 72, 156 (1982)

8) T. Yatagai, T. Nakadate, M. Idesawa and H. Saito, Opt. Eng. 21, 432 (1982)

9) T. Kreis, J. Opt. Soc. Am. 3, 6 (1986)

10) C.C. Huang, Opt. Eng. 23,365 (1984)

11) P. Hariharan, B.F. Oreb and Z. Wanzht, Opt. Acta 31, 989 (1984)

12) B. Dörband and H.j. Tiziani, Appl. Optics 24, 2604'(1985)

13) B. Dörband, Optik 60, 161 (1982)

14) F.M. Küchel and H.J. Tizfanf, Opt. Comm. 38, 17 (1981) 